\title{
Conversazione illustrata in Sicilia (1953): una controversia fra Vittorini e Crocenzi
}

\begin{abstract}
Originally published in 1941, Elio Vittorini’s Conversazione in Sicilia - probably his most important novel - was republished in 1953 with the inclusion of 169 photographs commissioned to Luigi Crocenzi three years earlier. This new publication was followed by a harsh controversy between the writer and the photographer regarding the authorship of the book. This essay recontructs the dispute - a crucial moment in the definition of Italian photographic culture - through the correspondence held in Vittorini's and Crocenzi's personal archives and the ensuing comments published in the media
\end{abstract}

\section{Keywords}

VITTORINI, ELIO; CROCENZI, LUIGI; CONVERSAZIONE IN SICILIA; PHOTOBOOK; NEOREALISM; 1950s; SICILY, LITTERATURE

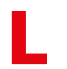

a settima edizione di Conversazione in Sicilia ${ }^{-1}$, romanzo centrale nell'opera di Elio Vittorini ${ }^{-2}$, uscì per Bompiani il 10 dicembre del 1953, illustrata con 188 fotografie, di cui 169 realizzate da Luigi Crocenzi ${ }^{-3}$. Alla pubblicazione seguì una vivace polemica tra Vittorini e Crocenzi in merito al riconoscimento del ruolo di autore delle immagini; una controversia che rappresenta una delle più significative vicende della cultura fotografica italiana del secondo dopoguerra.

Sebbene a posteriori Vittorini si sia sempre attribuito la paternità dell'idea, il volume era in realtà frutto di un'idea di Crocenzi che lo scrittore aveva accolto con grande interesse ${ }^{-4}$. I due si erano conosciuti nel dopoguerra, quando Vittorini aveva pubblicato su "Il Politecnico" alcuni lavori dell'allora giovanissimo fotografo marchigiano ${ }^{-5}$ ed era nata un'amicizia all’insegna della comune passione per il cinema e la 
fotografia. Difficile dire quando nacque in Crocenzi l'idea di illustrare Conversazione in Sicilia, ma è probabile che fu proprio in quegli anni, fra il 1946 e il 1947, che il fotografo iniziò a meditare sul progetto, intravedendo la possibilità di suggellare con le proprie immagini uno dei romanzi mito del neorealismo italiano. Nel 1949 Crocenzi propose a Vittorini di trarre dal libro anche un cortometraggio, presumibilmente da lui diretto: "si potrebbe abbinare il lavoro fotografico di illustrazione con uno cinematografico. Un cortometraggio sulla linea di 'Conversazione’ da lei sceneggiato" ${ }^{-6}$; lo scrittore, però, non prese mai nemmeno in considerazione questa idea, dichiarando anni dopo: "io non lascerei mai, per esempio, trarre un film da Conversazione, se non a patto d'esserne io stesso lo sceneggiatore e il regista" ${ }^{-7}$. Sebbene il volume sia uscito nel 1953, la realizzazione delle fotografie risale ai mesi di febbraio e marzo del 1950, quando Vittorini viaggiò appositamente in Sicilia con Crocenzi e quattro amici letterati: Vito Camerano, Alberto Cavallari, Giuseppe Grassi e Giovanni Pirelli. Tuttavia, gli esiti di quel viaggio non andarono come sperato da Vittorini che, rientrato a Milano e visti i provini di Crocenzi, rimase molto deluso dal lavoro del fotografo. In una lettera scritta al compagno di viaggio Giovanni Pirelli poco dopo il ritorno in città Vittorini si dichiarava:

depresso a causa delle fotografie. Sono quasi tutte non a fuoco. Quelle a fuoco non dicono niente di speciale. E le pochissime veramente buone sono le stesse che il nostro Luigi avrebbe potuto fare nel suo paese: bambine (che il diavolo se lo porti) e motivi di calligrafia capitolistica (dico capitolistica non capitalistica) ${ }^{-8}$.

Una disfatta che secondo Vittorini era prima di tutto tecnica:

lo al Luis [Luigi Crocenzi] non rimprovero il suo "stile". Allora avrei colpa io, perché sapevo già che cosa può dare. [...] $\mathrm{E}$ in fondo quello che non gli perdono è di non aver curato che le fotografie gli venissero a fuoco. Sono per l'80\% non a fuoco. Come se fosse un dilettante. Capisci questo? lo dico solo che non doveva sbagliare tecnicamente ${ }^{-9}$.

Sebbene già pochi giorni dopo Vittorini correggesse il tiro, ammettendo di avere "esagerato a proposito delle foto" e stimando in circa 200 quelle utilizzabili ${ }^{-10}$, rimase in lui un'incertezza di fondo sul lavoro che si protrasse per oltre due anni. Lo scrittore, però, si mosse fin da subito per cercare di rimediare al problema scrivendo, già nel marzo del 1950, al regista e fotografo Giacomo Pozzi Bellini, che verso la fine degli anni Trenta aveva realizzato diversi lavori in Sicilia, per chiedergli le fotografie di cui aveva bisogno per integrare il lavoro di Crocenzi. Per esse Vittorini offrì 500 lire, cifra giudicata “un po' bassa” da Pozzi Bellini, ma che si mostrò comunque accomodante, in virtù di altri progetti in corso tra i due ${ }^{-11}$. Il problema maggiore era, però, che Pozzi Bellini non disponeva di tutti soggetti richiesti da Vittorini ${ }^{-12}$. Sarà questa, con ogni 
probabilità, la ragione per cui, alla fine, verranno incluse nella selezione anche delle cartoline prive di autore.

Chi però, con viva acutezza, intuì già durante il viaggio quali erano state le difficoltà di interazione tra scrittore e fotografo, fu proprio Giovanni Pirelli, il quale li elencò a Vittorini con grande chiarezza in una lettera scritta poco dopo il rientro dalla Sicilia:

Quello che desidero dirti, e colla massima sincerità, è che mi pare che tu ti sia fatto delle illusioni. Da nessuno, capitato improvvisamente in Sicilia, potevi pretendere che la vedesse come tu l'avevi vista. La sola cosa che si poteva fare, e che fu fatta, era d'indicare i soggetti e di riprenderli in molti modi, sparando una quantità di foto. II Luis, scusami ma devo proprio difenderlo, riprese tutti i soggetti che tu gli indicavi. Che il lavoro fosse mal predisposto (che cioè si dovesse preventivamente elencare i soggetti "necessari" e spuntare a mano a mano che venivano fotografati); che tu vedessi ciò che volevi e non la fotografia di ciò che volevi e che Luis vedesse, viceversa, la fotografia ma non ciò che volevi (e ci sarebbe voluto ben altro allenamento nel lavoro comune, ben altra conoscenza da parte del fotografo del soggetto Sicilia, ben maggiore compenetrazione delle reciproche esigenze), tutto ciò mi pareva già palese nel corso del lavoro. Tu sei (posso dirlo?) alquanto egocentrico, nel senso che un discorso con te non è facile da iniziarsi se tu non lo richieda; e inoltre, in quel momento, l'importante era che lavoraste con fiducia, anche se la vostra grande fiducia a me pareva in buona parte ingenua. Che poi ci siano degli errori tecnici (di luce, di messa a fuoco) questo è molto spiacevole; ma sono convinto (e mi faccio forte anche del giudizio udito pronunciare da Albe S. [Steiner]) che nella fase di scoraggiamento che stai attraversando tu veda le cose più nere di quanto non lo siano ${ }^{-13}$.

La lettera di Pirelli, la cui sensibilità nel cogliere il rapporto tra scrittura e fotografia ne fa quasi un saggio sulla Conversazione in Sicilia illustrata in anticipo sui tempi, prosegue con altre osservazioni interessanti su aspetti che si riveleranno centrali nella polemica che scoppierà alla pubblicazione del libro, come quello dell'impaginazione:

Altro argomento che mi sta a cuore (e poi ho detto tutto e se mi picchierai ne avrai ben diritto). Tu ed il Luis avete sempre parlato di fare voi l'impaginazione. Vi ascoltavo con un misto di ammirazione e di sospetto. Questa è un'arte, un'arte a sé e difficilissima. Se tu ne fossi veramente capace direi che Vittorini è poeta e impaginatore [...]. Quanto al Luis mi pare abbia già un gusto sufficientemente formato e delle ottime possibilità; ma che sia oggi nelle condizioni di assolvere questo difficilissimo compito, ne dubito. Azzardo la mia conclusione: vi trovate di fronte a molto materiale, nel complesso buono, in parte ottimo; ma non avete la capacità di "vederlo" già trasformato nel volume ${ }^{-14}$. 
Il tema dell'utilizzo delle fotografie e della loro composizione sulla pagina rappresentava, quindi, un punto nodale, tanto che Pirelli prosegue:

lo proprio ti esorto, Elio, a mostrare il materiale a qualcuno che abbia già molta esperienza e se il suo giudizio fosse favorevole, di mettersi al lavoro con la collaborazione di quello. E quando il lavoro prendesse corpo e si vedesse che effettivamente mancano dei soggetti indispensabili, eventualmente rimandare il Luis in Sicilia, e che sia la Sicilia col gran sole d'estate!, a riprendere precisi soggetti. Tutta roba che costa, lo so. Ma mi pare che (parlando da capitalista) l'impegno sia già tanto grosso che è probabilmente più conveniente buttar dentro altri quattrini che non buttare a mare tutto ${ }^{-15}$

Pirelli conclude la lettera con la generosa proposta di contribuire alle spese del volume - offerta che Vittorini garbatamente e orgogliosamente declinerà ${ }^{16}$ - e con una critica a Crocenzi di non difendere "nei limiti in cui lo meriterebbe, il suo lavoro" ${ }^{-17}$.

Vittorini pare fare tesoro delle osservazioni dell'amico quando, a partire dal 1953, rimette mano al progetto, gestendo, in particolare, la questione dell'impaginazione, che avocherà completamente a sé escludendo del tutto Crocenzi: una scelta che sarà centrale nella discussione sull'autorialità del lavoro.

Il libro, infine, uscirà nel dicembre dello stesso anno con una nota di chiusura assai controversa che darà la stura alla vertenza tra scrittore e fotografo. Merita di essere riportata, almeno per la parte che a noi interessa:

è stato nel 1950, tredici anni dopo la comparsa della prima puntata di Conversazione sulla rivista "Letteratura" di Firenze, che sono tornato in Sicilia a fotografare, con l'aiuto non solo tecnico del mio amico Luigi Crocenzi, gran parte degli elementi di cui il libro s'intesse. Tranne dodici illustrazioni che ho ricavato da comuni cartoline, e sette fotografie prese nel 1938 da Giacomo Pozzi Bellini e da lui gentilmente prestate per questa pubblicazione, le altre, cioè 169 su 188, sono state colte tutte con la Leica e la Rolleiflex di Luigi Crocenzi nelle ultime settimane dell'inverno $1950{ }^{-{ }^{18}}$.

Crocenzi, che aveva passato gli ultimi mesi del 1953 a chiedere a Vittorini notizie sulla pubblicazione del volume ${ }^{-19}$, quando - all'inizio del 1954 - lo ha finalmente sottomano e legge la nota di chiusura, invia allo scrittore una lettera dal tono rabbioso e piena di rammarico:

Caro Elio, non puoi immaginare quanto sia rimasto male nel vedere in "Novità Bompiani" il libro presentato come "nuova edizione illustrata con centinaia di fotografie raccolte dall'autore", e io che ho fatto? E perché Bompiani mi ignora completamente? 
E perché i giornali parlano di Vittorini che è andato a fotografare con il mio aiuto?

Ma che aiuto? Giudico adesso la tua nota 1953 e adesso ne capisco la sottile ambiguità: "sono tornato a fotografare, con l'aiuto non solo tecnico (che significa?) del mio amico Luigi Crocenzi"... e poi... "sono state colte con la Leica e la Rolleiflex di Luigi Crocenzi". Allora chi avrebbe fatto le fotografie? Perché così il lettore può capire che Crocenzi avrebbe offerto le sue macchine, oppure ne avrebbe curato il trasporto da Milano in Sicilia. Insomma se io sono soltanto un "collaboratore" chi ha fatto le fotografie?

Tu hai forse bisogno di una fama di fotografo? -20

Alla missiva Crocenzi allega anche una lettera di diffida scritta dal suo avvocato, Luigi Dania di Porto S. Giorgio, e rivolta a Valentino Bompiani; qualora Vittorini non dia risposta entro 48 ore chiarendo le sue intenzioni future in merito alla vicenda, ammonisce Crocenzi, la diffida verrà inviata all'editore. Essa riporta in tutto e per tutto una lettera che il fotografo aveva inviato personalmente a Bompiani pochi giorni prima senza ricevere risposta alcuna ${ }^{-21}$ (a questo si riferiva la frase "perché Bompiani mi ignora completamente?”). Bompiani aveva infatti ritenuto di dover ignorare Crocenzi fino al punto di non premurarsi nemmeno di spedirgli una copia omaggio del volume, atteggiamento che la dice lunga su come venisse inteso il ruolo del fotografo nell'ambiente editoriale di quegli anni.

Crocenzi, dunque, per tramite dell'avvocato Dania, lamenta di non avere ricevuto le copie omaggio che gli spettavano, di non avere avuto corrisposti i diritti d'autore per le fotografie, di aver lasciato intendere che le fotografie le avesse fatte Vittorini e, infine, di avere completamente omesso il nome del fotografo nel lancio pubblicitario del volume. La richiesta che viene quindi fatta a Bompiani, per "evitare un'azione legale nei confronti Suoi e del Vittorini”, è di "regolarizzare con il Crocenzi la questione dei diritti d'autore" e soprattutto

di precisare con un comunicato stampa e con una nota ben evidente nel suo prossimo bollettino editoriale (o possibilmente con un dépliant da allegare in "Conversazione in Sicilia") che le 169 fotografie sono state eseguite unicamente dal sig. Luigi Crocenzi ${ }^{22}$.

Con ogni evidenza Vittorini non soddisfa la richiesta di chiarimento fattagli da Crocenzi. Parte quindi la lettera dell'avvocato Dania per Bompiani, fatto che genera la reazione indignata di Vittorini, il quale invia una serie di lettere all'editore, al fotografo e all'avvocato, nelle quali ribadisce la sua versione dei fatti. A Bompiani, il 18 gennaio 1954, scrive:

Caro Valentino, ho letto la lettera dell'avvocato Diana [sic!] e sono più sorpreso di te. Non ti ho mai parlato di diritti delle fotografie, perché 
non ce n'erano. Le fotografie furono fatte completamente a mie spese, compreso il viaggio e il soggiorno del Crocenzi in Sicilia con me, e il Crocenzi ha sempre dichiarato, anche di fronte a testimoni (come del resto era pacifico sin dal giorno della partenza) di ritenersi pienamente retribuito dal rimborso delle spese e dalla "sperata pubblicazione". Tanto egli teneva a ciò che ora ha ottenuto, ossia alla pubblicazione, da aggiungere che era disposto a concorrere alle spese di edizione o ad interessare le case produttrici del materiale fotografico, affinché esse potessero contribuire ${ }^{-23}$.

$$
\text { - }
$$

Le affermazioni di Vittorini trovano riscontro solo nel fatto che era stato lui, effettivamente, a pagare il viaggio di Crocenzi, dato che questi in una lettera del 19 gennaio 1950, poco prima della partenza per la Sicilia, dichiarava di non essere "assolutamente in grado di sopperire alle spese per il viaggio e la attrezzatura tecnica”. Tuttavia, in quella stessa lettera, Crocenzi chiedeva anche: "poi nella edizione del volume avrò una percentuale?” - ${ }^{24}$. Se Crocenzi abbia poi deciso di rinunciare ai diritti, così come che si sia offerto di contribuire ai costi di edizione del volume, fu questione del tutto verbale di cui non risulta traccia alcuna negli archivi consultati ${ }^{25}$. Ha però un riscontro il fatto che Crocenzi propose di coinvolgere come sponsor le ditte fotografiche delle quali si era servito - Ferrania, Gevaert e Kodak per le pellicole, Leica e Rolleiflex per le fotocamere ${ }^{-26}$ - ma non essendoci stata risposta da parte di Vittorini è probabile che fu proprio lo scrittore a non essere interessato a perlustrare le possibilità di questa proposta.

La lettera di Vittorini a Bompiani prosegue con alcune indicazioni che sono molto utili per comprendere le ragioni dello scrittore e la sua concezione del ruolo del fotografo, assai riduttiva forse perché subordinata alla sua regia, così come aveva messo in luce l'amico Pirelli che, in Sicilia, era stato testimone del lavoro condotto dai due:

Aggiungo che il Crocenzi si è limitato soltanto a scattare le fotografie dei luoghi, delle cose e delle persone che gli indicavo volta a volta, suggerendogli talora anche i punti di vista da cui fotografarli, e costringendolo a ripetere perfino cinque-sei volte lo stesso soggetto. Dalla massa di 1620 negativi ho scelto quindi io (spesso con tagli che riducevano la foto a un più piccolo particolare) le 188 fotografie che illustrano il volume.

Non capisco proprio che cosa voglia il Crocenzi. Il rilievo che gli ho dato nel volume parlando di collaborazione "non solo tecnica" è in realtà superiore alla parte da lui svolta ${ }^{27}$.

Per Vittorini, insomma, "è evidente che il Crocenzi vuol fare solo un po' di rumore" ${ }^{\mathbf{2 8}}$. Nella lettera che, invece, lo scrittore invia a Crocenzi, oltre a dichiararsi "amareggiato e offeso dallo sviluppo della grana" -29, espone con una serie di cifre e dati quali sono stati i reali costi del volume, dichiarando di trovarsi, al momento, "scoperto per più di 
200.000 lire" ${ }^{30}$. Spiega che, dunque, qualora Crocenzi volesse una percentuale degli utili, dovrebbe prima partecipare alle spese. Vittorini parla anche dell'esecuzione "di ciascuna delle foto stampate, quella tale esecuzione che io ho chiamata nella mia nota 'non solo tecnica' e che cioè ho riconosciuta anche come 'artistica"” $-{ }^{31}$. Si tratta, tuttavia, di un riconoscimento implicito che appare inadeguato e di comodo da parte di Vittorini, per di più confinato nell'ambito di una lettera privata (a Bompiani aveva invece detto l'esatto contrario). A Crocenzi, infatti, non basterà.

In una minuta manoscritta, poi non spedita, Vittorini mostrava un'amarezza ancora maggiore verso Crocenzi:

\begin{abstract}
$-$
Non capisco che cosa ti abbia mai fatto perché tu mi faccia passare cosi di umiliazione in umiliazione. Debbo pensare che tu mi odiassi già prima di conoscermi e che ti sia finto mio amico per attirarmi in un tranello? Sono l'unica persona che ti ha allungato una mano (fin dai tempi di "Politecnico") e tu questa mano me la mordi. La tua ingratitudine è cosi enorme che inizio a pensarla come una malattia e a compatirla -32 .
\end{abstract}

I sentimenti di offesa e riprovazione di Vittorini emergono qua con chiarezza e spiegano perché, anche anni dopo, non sarà possibile arrivare a una vera conciliazione tra i due.

La lettera più interessante è, però, quella che Vittorini scrive all'avvocato Dania, nella quale ribadisce le cose dette a Bompiani riguardo al fatto che Crocenzi abbia più volte espresso una rinuncia a eventuali diritti d'autore. Aggiunge, però, al fine di chiarire meglio le cose, alcuni punti di sicuro rilievo:

Ora io non so che cosa il Crocenzi Le abbia detto, che cosa abbia dimenticato e che cosa sia accaduto, ma le cose stanno nel modo che qui di seguito Le espongo.

1) Le fotografie (a parte quelle di Giacomo Pozzi Bellini e le anonime) sono state scattate dal signor Luigi Crocenzi.

2) L'autore del libro si è limitato a svolgere, per tali fotografie, un lavoro di tipo registico scegliendo i luoghi dove prendere le fotografie stesse, e indicando volta a volta, in generale, al signor Crocenzi settori di paesaggio, cose e persone da fotografare.

3) Si aggiunge, a completare la precisazione, che il rimanente lavoro illustrativo dell'autore del testo è stato del tipo che in linguaggio cinematografico vien detto di montaggio, ossia il lavoro per cui, da una massa di un migliaio di negativi, sono state scelte (spesso con tagli che riducevano la foto a un suo piccolo particolare) e ordinate nell'attuale successione, le 188 fotografie che illustrano il volume ${ }^{-33}$.

$-$

In queste poche righe si trova espressa, con concisione pari alla precisione, l'idea che Vittorini aveva di tutto il lavoro per la Conversazione 
illustrata. Si può innanzitutto notare con che opportunismo Vittorini dichiarasse a Bompiani che era Crocenzi quello che si era "limitato soltanto a scattare le fotografie”, mentre all'avvocato Dania dichiara che è stato lui, in quanto autore del testo, quello che si è "limitato a svolgere [...] un lavoro di tipo registico". Insomma, nello sminuire prima il fotografo e poi sé stesso, a seconda dell'interlocutore, è difficile non vedere un pizzico di malafede da parte di Vittorini. Tanto più nel fatto che, per la prima volta, egli riconosceva finalmente la paternità di Crocenzi sulle fotografie, dopo che per anni aveva raccontato di averle fatte lui ${ }^{-34}$.

Ben più di sostanza è, però, la questione "registica" che illustra bene il metodo di lavoro di Vittorini e fa chiarezza sul perché lui ritenesse il fotografo un mero esecutore, un tecnico addetto a premere il pulsante di scatto e nulla più. Vittorini, infatti, si approccia al libro illustrato con modi e termini mutuati direttamente dal cinema. Così il fotografo diventa un operatore e, nelle idee di Vittorini, è padrone e autore delle immagini quanto un operatore cinematografico lo è di un film, cioè niente o giù di lì. Questo pensiero è espresso in forma compiuta in un celebre articolo pubblicato nell'aprile del 1954 su "Cinema Nuovo" -35 nel quale Vittorini ricostruisce il suo rapporto con le immagini a partire da Americana, passando a "Il Politecnico", per arrivare infine all'edizione illustrata di Conversazione in Sicilia. Traspare in questo scritto un palese disprezzo per i fotografi, ridotti a meri produttori di icone ai quali non riconoscere la benché minima capacità espressiva. Parlando di Americana, infatti, Vittorini afferma:

A me non importava nulla del valore estetico o illustrativo che la fotografia poteva avere singolarmente ciascuna per sé. M'interessava solo che ogni fotografia avesse un suo contenuto materiale (che cioè riproducesse un certo "oggetto"), e procedevo alla scelta delle fotografie proprio come avrei potuto scegliere, presso dei rigattieri, gli oggetti di cui ammobiliare una stanza, senza minimamente badare a provenienze, qualità, tecniche e pretese di stile ${ }^{-36}$.

$$
\text { - }
$$

La funzione delle fotografie era quella di prestarsi, tramite il loro accostamento e la loro successione, alla creazione di un senso che era quello voluto e inteso da Vittorini. Un lavoro, appunto, di tipo registico, che sarà il metodo seguito anche per Conversazione in Sicilia. Resta il dubbio se questo tipo di lavoro fosse davvero così inconciliabile con il riconoscimento di quello dei fotografi che avevano realizzato le immagini: Crocenzi per Conversazione e i grandi maestri del modernismo statunitense per Americana. Probabilmente coglieva nel segno Giovanni Pirelli quando scriveva che la ragione dei problemi sorti con Crocenzi in Sicilia era data dal fatto che lo scrittore fosse "alquanto egocentrico" o, perlomeno, secondo le parole di Giovanni Falaschi, affetto da "reticenze abituali" e da una tendenza a scrivere sempre una sorta di "autobiografia ideale" ${ }^{37}$. Vittorini, quasi a conferma di questo, prosegue l'articolo per "Cinema Nuovo" scrivendo candidamente che del libro illustrato 
lui voleva essere "anche il principale esecutore. Non volevo correre il rischio di trovarmi il libro ingombrato da una 'interpretazione' che gli si sovrapponesse e ne ostacolasse, con delle pretese autonome, la lettura" ${ }^{38}$. Per questo motivo, spiega Vittorini, si rifiutò di scrivere un "treatment" - ${ }^{39}$, come invece gli aveva chiesto Crocenzi prima della partenza per la Sicilia ${ }^{-40}$, ma preferì invece assicurarsi "la passività del fotografo"-41. Vittorini si appoggia alla definizione di "film immobile" -42 per spiegare il suo specifico uso della fotografia nell'illustrazione di un romanzo. Conversazione in Sicilia è, d'altronde, un romanzo dallo stile molto visivo, con scene che ricordano spesso una cartolina vera e propria, in una concezione "intermediale" della letteratura ${ }^{-43}$ per la quale l'edizione illustrata dell'opera appare quasi come uno sbocco naturale. C'è perfino chi, come Riccardo Paterlini, si è spinto a dire che già la prima edizione del romanzo, quella del 1941, conteneva in nuce una traccia fotografica ${ }^{44}$.

Verso la fine del gennaio 1954, la polemica tra Vittorini e Crocenzi tracima sui giornali, di pari passo con le recensioni (invero non troppo entusiaste) ${ }^{-45}$ che accompagnano la pubblicazione; è Crocenzi a essere attivissimo nello scrivere lettere di precisazione tese a evidenziare il suo ruolo di co-autore, permettendosi anche lo sfregio di imputare la tiepida ricezione del libro proprio agli errori di Vittorini nel trattamento delle fotografie. In una lettera scritta ad Angelo del Boca, in risposta a un articolo che questi aveva pubblicato su "Gazzetta sera" del 29 gennaio 1954, Crocenzi racconta la sua versione del viaggio in Sicilia:
Quando Vittorini voleva "fotografare" qualcosa io non ero pronto, mi impasticciavo e non "folgoravo" niente oppure fotografavo male. $\mathrm{E}$ male non solo perché io non sono un operatore ubbidiente [...] o per- ché il cielo della Sicilia fu in quei giorni maledettamente dispettoso, fotografavo male perché il mio stile e le mie idee mi imponevano di fare a modo mio. Non collaborai. Perché in me era un'altra visione del- le cose, un guardare, un incantarsi su altro, una ambizione di cercare le "mie" immagini ${ }^{46}$.

In questa discordanza di intenti, già lucidamente descritta da Giovanni Pirelli quattro anni prima, risiedeva, secondo Crocenzi, il motivo della delusione di Vittorini trovatosi al cospetto delle immagini:

Le fotografie mie non piacquero, altre idee aveva in mente lui per il suo libro, parve gli mancassero tutte le foto necessarie, e lui le voleva, e montarono in lui grandi e non astratti furori. Cosi Conversazione rimase per lunghi anni in attesa, finché Vittorini cominciò a capire e sentire tanto le mie fotografie da poterle impaginare -47 .

$$
-
$$

Conclude Crocenzi che "fu lì dunque il guasto, invece di 'scattare' io 'feci' le mie fotografie" -48. Intervistato per "La notte", Vittorini, dal canto suo, ribadisce il suo concetto di film immobile e sostiene a più 
riprese di avere dato al lavoro di Crocenzi il giusto rilievo: "forse che si mette sul frontespizio di un libro in 'Bodoni nero 16' il nome di una persona che ha fatto semplicemente il 'facchino'?” -49. Vittorini manifesta fastidio anche per il fatto che Crocenzi abbia fatto circolare per le redazioni i loro scambi epistolari ${ }^{-50}$ ma chiarisce che "io posso ancora pubblicare il libro senza illustrazioni e non il fotografo senza il testo" ${ }^{\mathbf{5 1}}$. Che è proprio quello che succederà nei decenni successivi $\mathbf{- 5 2}^{\mathbf{5 2}}$.

Vittorini, rispetto a Crocenzi, può giocare con una maggiore confidenza all'interno degli ambienti letterari. Ecco così i critici accorrere in suo soccorso, come nel caso di Enrico Falqui, autore di una recensione a Conversazione uscita su "Tempo" il 26 gennaio del $1954^{-53}$, che in privato scrive sussiegoso a Vittorini: "non sarò io a dare spago agli invasati sul tipo del Crocenzi" - ${ }^{54}$. Purtroppo per lui gli toccherà, invece, dargli spago tramite una precisazione che sarà pubblicata il 12 marzo ${ }^{-55}$. Il giorno prima, Falqui si premura di avvisare Vittorini della cosa, rassicurandolo che la noticella verrà pubblicata solo per obbligo di legge $\mathrm{e}$ commentando: "Ma codesto Crocenzi si deve reputare un eccelso artista, il che risulta forse un tantino esagerato no?" ${ }^{-56}$; ma probabilmente anche il Falqui si sopravvalutava nel reputarsi un buon critico, se non era in grado di capire le ragioni per cui Crocenzi difendeva il proprio lavoro. Oppure Giuseppe Cintioli, che scrive una lettera a Vittorini per esprimere le sue impressioni, per così dire, "a caldo", con il libro appena preso in mano ${ }^{-57}$ e poi una più articolata recensione su "Comunità" ${ }^{58}$. Ebbene Cintioli riesce nella non facile impresa, tra lettera e articolo, di non fare mai nemmeno una volta il nome di Crocenzi; lunghe analisi delle fotografie, del loro significato, della loro forza o debolezza, prescindono completamente dallidea che queste siano state prodotte da un fotografo e che con esso, almeno in parte, ci si debba confrontare. "Milano sera" pubblica, invece, un breve articolo che racconta per sommi capi la vicenda per poi mettere in dubbio la legittimità della pretesa di riconoscimento di Crocenzi, dato che era stato Vittorini a curare l'impaginato e a indicare i soggetti da fotografare ${ }^{-59}$; a destare sospetto, in questo caso, è il fatto che su quel quotidiano scriveva abitualmente come critico cinematografico Giusto Vittorini, il figlio di Elio.

Sono solo pochi esempi tra i tanti possibili, ma nella cronaca del tempo Crocenzi è effettivamente bistrattato, trattato alla stregua di un mitomane in cerca di facile e immeritata pubblicità. Si tratta di un pregiudizio assai diffuso tra gli italianisti, che a lungo ha accompagnato, e ancora oggi accompagna, l'edizione illustrata di Conversazione in Sicilia. Basti dire che un critico intelligente come Giuseppe Lupo, in una "controstoria di una polemica”, dopo un'acuta analisi della Conversazione illustrata, arriva a sostenere che "in realtà non è difficile giudicare come pretestuoso il carattere delle richieste di Crocenzi (che chiede diritti d'autore e visibilità pubblicitaria)" ${ }^{60}$. Non si capisce perché, invece, la maniacale attenzione prestata da Vittorini ai compensi (come testimoniato dalle carte con Bompiani) e al consenso (come testimoniato dal promiscuo rapporto con i critici) non sia invece passibile di altrettanta riprovazione. 
Epifanio Ajello, invece, non mostra alcun dubbio: il "principale esecutore" delle immagini è Vittorini ed è con lui che ci si deve confrontare per l'analisi del volume, arrivando perfino a domandarsi come "fotografa" Vittorini, intendendo con ciò il modo in cui tratta le fotografie ${ }^{-61}$.

Anche Margherita di Fazio è dello stesso avviso e in un suo studio dedicato ai rapporti tra fotografia e scrittura analizza la Conversazione illustrata come opera del solo Vittorini. In apertura dello scritto, la studiosa ci dice che Vittorini "intraprese un viaggio in Sicilia nel 1950 con il fotografo Crocenzi” e sarà l'unico riferimento del saggio all'esistenza di un fotografo ${ }^{-62}$.

Paolo Orvieto supera tutti di slancio quando, andando a ritroso nella genesi del volume, parla di Andiamo in processione, il lavoro di Crocenzi pubblicato su "Il Politecnico" nel 1947. Orvieto non solo ritiene che le didascalie del racconto fotografico siano di Vittorini, ma che anche l'idea stessa e il soggetto vadano considerate opera dello scrittore ${ }^{-63}$. Implicitamente, si lascia così intendere che anche questo lavoro sia da attribuire a Vittorini:

la fotografia dunque non deve essere un'espressione artistica autonoma (non può costituirsi come arte a sé stante): illustra, con criterio "cinematografico" e non "fotografico", in una "cronaca" passibile di ogni dilatazione (ma sempre per merito della narrazione) i concetti; concetti che, mai elusi o traditi, continuano a incombere nell'immagine determinandone il valore e lo stesso processo ermeneutico ${ }^{-64}$.

Va da sé che, in quest'ottica, anche la Conversazione illustrata sarebbe da ritenere integralmente opera di Vittorini.

A cercare di andare oltre sono Bart Van den Bossche e Jan Baetens, che insistono molto sull'opera di montaggio e selezione fatta da Vittorini come elemento autoriale, ma che non escludono dall'analisi e dalla valutazione il lavoro di Crocenzi. I due studiosi belgi riescono a distinguere le due parti con chiarezza e sebbene, in quanto italianisti, il loro focus sia l'opera Vittorini, hanno ben presente che l'edizione illustrata della Conversazione fu possibile anche grazie alla partecipazione di un fotografo ${ }^{-65}$.

E un approccio non dissimile da quello di Maria Rizzarelli. La studiosa, infatti, si sofferma a lungo sul ruolo giocato dagli inserti testuali e dalle didascalie nel definire il significato delle immagini e nel contestualizzarle nel flusso narrativo ${ }^{-66}$, ma riconosce anche la capacità delle fotografie di parlare da sole attraverso il loro specifico linguaggio ${ }^{-67}$.

La disputa tra scrittore e fotografo andrà avanti ancora per oltre un anno e, alla fine, si risolverà per via extragiudiziale nell'estate del 1955 con un accordo del quale a oggi rimangono ignote le condizioni ${ }^{68}$. Il rapporto tra i due si ricomporrà lentamente anche grazie all'opera di Raul Lunardi, scrittore di origini marchigiane come Crocenzi-69. In una lettera del luglio 1955 Vittorini scriverà a Lunardi: 
Ricambia pure i saluti a Crocenzi - Ma io vorrei vederlo e dargli una risciacquata dopo la grana che mi ha fatto avere l'anno scorso con Bompiani. Rancore non gliene porto, però mi piacerebbe avere uno sfogo a voce con lui. Se vogliamo tornare amici bisogna bene che ci si incontri e ci si spieghi. Non si può mica fare finta di nulla così ${ }^{70}$.

Negli anni seguenti i due riallacciarono i contatti, anche se in maniera distaccata. In particolare, fu Crocenzi a cercare di coinvolgere Vittorini in varie iniziative, alle quali lo scrittore rispose sempre con tono un po' freddo, passando definitivamente dal tu al lei ${ }^{-71}$. Crocenzi continuò a cercare di realizzare progetti di adattamenti letterari o reportage di carattere etnologico, sulla falsariga di come lui aveva inteso la Conversazione, affiancando a essi anche una continua riflessione teorica all'interno del Centro per la Cultura Fotografica che aveva fondato a Fermo sul finire del 1954. Nell'ambito delle iniziative del Centro, Crocenzi ripubblicò lo scritto di Vittorini apparso su "Cinema Nuovo", previa autorizzazione dell'autore il quale aveva rifiutato di scrivere un nuovo articolo per l'occasione ${ }^{-72}$. Crocenzi riadatterà quell'articolo in forma di opuscoletto, espunto però delle parti in cui lo scrittore polemizzava sulla vertenza ${ }^{-73}$, sottoponendolo a Vittorini per la correzione e l'approvazione definitiva ${ }^{-74}$.

Tra le tante iniziative di Crocenzi, forse la più ambiziosa, e naufragata già prima di salpare, fu quella di una collana di fotolibri dedicati all'Italia da pubblicare con Bompiani; un'idea che Vittorini, nel 1956, pur non disdegnandola affatto, ritenne non fattibile, oltre che per motivi economici forse anche per sfiducia verso Crocenzi e il gruppo del Centro per la Cultura Fotografica.

Da tempo si era pensato a dei "Gettoni" fotografici. Nel momento addirittura in cui la collana di letteratura ebbe inizio. Dovevano alternarsi con i "Gettoni" di narrativa. Purtroppo la cosa, comportando una spesa considerevole da parte della Casa Editrice, non si realizzò. E non credo che possa realizzarsi oggi che esiste già una collana fotografica presso Einaudi, I'“'Italia mia". Ne parlerò comunque all'Editore e, più avanti, vedremo ${ }^{-75}$.

Italia mia era la collana presso la quale venne pubblicato nel 1955 , con il numero uno, il celebre fotolibro Un paese di Paul Strand e Cesare Zavattini ${ }^{-76}$. La collana Einaudi, però, non ebbe mai un numero due e neanche i "Gettoni fotografici" per Bompiani decollarono mai, segno di un interesse diffuso verso i fotolibri da parte degli addetti ai lavori che, però, nell'Italia di quegli anni, si scontrò con insormontabili ragioni di tipo economico ${ }^{-77}$.

La vicenda di Conversazione in Sicilia ricorda in parte anche un'altra celebre contesa di qualche anno posteriore, quella tra Franco Pinna ed Ernesto De Martino che fece seguito alla pubblicazione, nel 1961, de 
La terra del rimorso ${ }^{-78}$, con numerose fotografie delle quali è pressoché assente il riconoscimento nell'edizione. Tuttavia questa vicenda è diversa sotto molti aspetti, a partire dal fatto che quello di De Martino non può certo essere considerato un fotolibro ma è, piuttosto, un'indagine scientifica. In questo percorso di ricerca, il valore delle immagini, e con esse il ruolo del fotografo, può considerarsi "del tutto marginale", come spiegato bene da Lello Mazzacane ${ }^{-79}$ in riferimento all'Atlante figurato del pianto ${ }^{-80}$. D'altronde, l'autonomia intellettuale di Pinna era ben altra da quella di Crocenzi. Mentre quest'ultimo doveva seguire le direttive di Vittorini, Pinna era libero di assecondare le proprie inclinazioni e realizzava, al di fuori del lavoro con De Martino, anche fotografie per proprio uso personale ${ }^{-81}$.

Nell'archivio di Luigi Crocenzi c'è un foglio manoscritto, privo di indicazioni sulla data, ma presumibilmente posteriore di molti anni alla Conversazione, in cui Crocenzi rievoca quel lavoro e annota:

Ricordo che io avevo una mia tesi diversa da quella di Vittorini. Sì, le foto entrassero pure nelle colonne delle parole ma che potessero anche concatenarsi fra loro, dialogare si con le parole ma essere una nuova spinta narrativa per il montaggio visivo sulla pagina, una serie staccata di sequenze, anche.

Prevalse, giustamente, la tesi di Vittorini ${ }^{\mathbf{8 2}}$.

Per Raffaella Turrin "non è certo se quel 'giustamente' sia una consapevole dichiarazione poetica $\mathrm{o}$, al contrario, costituisca in qualche modo un doveroso tributo intellettuale alle scelte di uno scrittore di grande spessore ed esperienza" ${ }^{83}$. Di sicuro è, a distanza di anni, un ulteriore segno della difficoltà di valutare, anche da parte dei protagonisti, la complessità di un lavoro che segnò la conclusione simbolica del neorealismo italiano, attraverso "il mancato riconoscimento del primo libro fotografico realizzato nel nostro paese" ${ }^{84}$.

\footnotetext{
$-{ }^{1}$ Vittorini 1953b.

La prima edizione del romanzo uscì nel 1941 per l'editore Parenti di Firenze col titolo Nome e lagrime. A partire dalla seconda edizione (Milano, Bompiani, 1941) verrà adottato il titolo, originariamente voluto da Vittorini, Conversazione in Sicilia. Un'altra edizione del volume uscì nel 1973, diversi anni dopo la
}

morte di Vittorini, come libro strenna Olivetti con le fotografie di Enzo Ragazzini (cfr. Pontillo 2018).

- 2 Sull'opera di Elio Vittorini (1908-1966) la bibliografia è vastissima. Ci limitiamo a rimandare a Lupo 2011 per un'accurata analisi del rapporto che lo scrittore ebbe con la cultura visuale.

\author{
$-{ }^{3}$ Le restanti 19 sono 12 \\ cartoline senza indicazione \\ dell'autore e 7 fotografie \\ realizzate da Giacomo \\ Pozzi Bellini nel 1939. \\ $-{ }^{4}$ Vittorini 1950b, p. 311. \\ -5 Sulla figura di Luigi \\ Crocenzi (1923-1984) si \\ vedano principalmente: \\ Zannier 1996; \\ Amodeo / Giusa / Turrin \\ 2003; Liva 2011; \\ sull'archivio di Luigi \\ Crocenzi conservato al
}


CRAF di Spilimbergo si veda Liva 2019; per i lavori pubblicati su "II Politecnico" cfr. Crocenzi 1946a, Crocenzi 1946b

e Crocenzi 1947.

- 6 \#Crocenzi 1949.

- 7 In Vittorini 1954, p. 202.

${ }^{-}{ }^{8}$ Vittorini 1950a, p. 309.

Corsivi nell'originale.

$-{ }^{9}$ Vittorini 1950b, p. 311.

Corsivi nell'originale.

$-{ }^{10}$ Ivi, p. 312. Si tratta

di una postilla alla lettera, datata 3 aprile 1950.

- 11\#Pozzi Bellini 1950a.

Pozzi Bellini stava

attraversando un periodo di difficoltà economica e, per tramite di Vittorini, gli si prospettava la possibilità di un contratto a Milano presso Mondadori.

- 12 \#Pozzi Bellini 1950b.

- 13 \#Pirelli 1950.

-14 Ibid.

- 15 Ibid. Sottolineatura nell'originale.

- ${ }^{16}$ Vittorini 1950b, p. 311.

- 17 \#Pirelli 1950.

- 18 Vittorini 1953b,

p. 225.

- ${ }^{19}$ Crocenzi scrive a

Vittorini per chiedere

della pubblicazione di

Conversazione in Sicilia il

21 settembre, il 21 ottobre,

il 13 novembre, il 25

novembre, il 18 dicembre

1953 e ancora il 10 gennaio

1954, a volume ormai

uscito: “Vito [Camerano]

mi scrisse che l'editore

mi avrebbe spedito

copie del libro, ma finora

non ho ricevuto niente"

(\#Crocenzi 1954a).

- 20 \#Crocenzi 1954c.

- 21 \#Crocenzi 1954b.

- 22 \#Dania 1954.

- 23 \#Vittorini 1954a.

- 24 \#Crocenzi 1950.

- 25 “Egli [Crocenzi] non

è un povero diavolo che

vive del suo lavoro. Ė un

signore di provincia che

vive delle rendite che gli

passa la famiglia. lo ho commesso l'errore di non fargli mettere per iscritto tutto questo che mi ha ripetutamente assicurato" (\#Vittorini 1954e)

- 26 \#Crocenzi 1953.

- 27 \#Vittorini 1954a.

Come accennato, le

fotografie di Crocenzi

erano in effetti 169.

-28 lbid.

- 29 \# Vittorini 1954b

- 30 lbid.

-31 lbid.

- 32 \#Vittorini 1954c.

- 33 \#Vittorini 1954d.

- 34 “Tra qualche mese

uscirà un'edizione di

Conversazione con

duecento e più fotografie

che ho prese allo scopo

in Sicilia con uno del

mestiere due anni fa"

(Vittorini 1952, p. 53);

“Sta per uscire presso

Bompiani un'edizione

di Conversazione con

fotografie prese da me e

un mio amico" (Vittorini

1953a, p. 123)

-35 Vittorini 1954,

pp. 200-202.

- 36 Ivi, p. 200.

- 37 Falaschi 1987, p. 37.

- 38 Vittorini 1954, p. 201.

- 39 lbid.

- 40 \#Crocenzi 1950.

- 41 Vittorini 1954, p. 201.

-42 Ivi, p. 200.

- 43 Brohm 2007.

- 44 Paterlini 2014, p. 135.

- 45 Rizzarelli 2007,

pp. 20-21.

- 46 \#Crocenzi 1954d.

-47 Ibid.

- 48 Ibid.

- 49 Crovi 1954.

- 50 Amara per Vittorini

1954.

- 51 Crovi 1954.

- 52 Solo nel 2007 uscirà una ristampa anastatica del volume, a cura di Maria Rizzarelli (Milano, RCS).

- 53 Falqui 1954.

- 54 \#Falqui 1954a.

- 55 II fotografo poeta

1954.
- 56 \#Falqui 1954b.

- 67 \#Cintioli 1954.

- 58 Cintioli 1954.

- 59 G.P. 1954.

- 60 Lupo 2011, p. 91.

Storia e controstoria di una polemica è il titolo del paragrafo che tratta l'argomento (pp. 87-95).

- 61 Ajello 2008,

pp. 166-167.

- 62 Di Fazio 2005, p. 94.

- 63 Orvieto 2005, p. 73.

-64 Ibid.

- 65 Van den Bossche /

Baetens 2015.

- 66 Rizzarelli 2016,

pp. 209-211.

-67 Ivi, p. 212.

- 68 Russo 2011, p. 37.

- 69 Lupo 2011, p. 94.

- 70 Vittorini 1955, p. 289.

- ${ }^{71}$ Le carte dell'archivio

Crocenzi presso il CRAF

di Spilimbergo conservano diverse lettere tra i due che arrivano fino all'ottobre del 1963.

- 72 “Caro Crocenzi,

mi dispiace, ma non ho

tempo e non mi sento,

per la verità, di scrivere

l'articolo che mi chiede.

Se lei crede di poter trarre

da quel mio intervento

in 'Cinema Nuovo' tutti i

passi che le potrebbero

interessare, faccia pure"

(\#Vittorini 1956b).

- 73 \#Nuove esperienze

1957.

- 74 \#Vittorini 1957.

- 75 \#Vittorini 1956a.

- 76 Strand / Zavattini

1955.

- 77 Vittorini aggiunge

in calce alla sua lettera

questa nota manoscritta:

“il problema è di poter

contenere le spese entro

limiti tali da mettere i libri

a prezzi non cari: e non si

riesce, per quanto si cerchi,

a risolverlo" (\#Vittorini

1956a).

- 78 De Martino 1961.

- 79 Mazzacane 1996,

p. 129. 
-80 Si tratta di una appendice a De Martino 1958.

\author{
- 81 Cfr. Faeta 1999, \\ pp. 76-77 e Mazzacane \\ 1996, pp. 132-133.
}

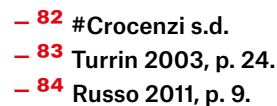

Ajello 2008 Epifanio Ajello, Il racconto delle immagini. La fotografia nella modernità letteraria italiana, Pisa, ETS, 2008.

Amara per Vittorini 1954 Amara per Vittorini la settima Conversazione in Sicilia, in "La Notte", 30 gennaio 1954.

Amodeo / Giusa / Turrin 2003 Fabio Amodeo / Antonio Giusa / Raffaella Turrin (a cura di), Luigi Crocenzi. Un racconto per immagini, Spilimbergo, CRAF, 2003.

Brohm 2007 Heke Brohm, Elio Vittorini e l'intermedialità. A proposito di Conversazione in Sicilia del 1953, in "Rivista di letteratura italiana", n. 2, 2007, pp. 87-104.

Camerano / Crovi / Grasso 2006 Vito Camerano / Raffaele Crovi / Giuseppe Grasso (a cura di), La storia dei Gettoni di ElioVittorini, Torino, Aragno, 2006, 3 voll.

Cintioli 1954 Giuseppe Cintioli, Testo e immagini, in “Comunità", a. VIII, n. 24, aprile 1954, pp. 68-70.

Crocenzi 1946a Italia senza tempo, fotografie di Luigi Crocenzi, in "II Politecnico", n. 28, 6 aprile 1946, p. 2 (servizio di 8 fotografie).

Crocenzi 1946b Luigi Crocenzi, Occhio su Milano, in "Il Politecnico", n. 29, 1 maggio 1946, pp. 13-15 (servizio di 19 fotografie).

Crocenzi 1947 Andiamo in processione, racconto fotografico di Luigi Crocenzi, in “Il Politecnico", n. 35, gennaio/marzo 1947, pp. 54-59 (servizio di 34 fotografie).

Crovi 1954 Raffaele Crovi, Fotografi e no, in "La Notte”, 30 gennaio 1954.

De Martino 1958 Ernesto De Martino, Morte e pianto rituale nel mondo antico. Dal lamento pagano al pianto di Maria, Torino, Einaudi, 1958.

De Martino 1961 Ernesto De Martino, La terra del rimorso. Contributo a una storia religiosa del Sud, Milano, II Saggiatore, 1961.

Di Fazio 2005 Margherita di Fazio, Scrittura e fotografia. Fotografia e scrittura. Due percorsi a confronto, in Bruna Donatelli (a cura di), Bianco e nero. Nero su bianco. Tra fotografia e scrittura, Napoli, Liguori, 2005, pp. 93-108.

Faeta 1999 Francesco Faeta, Dal paese al labirinto. Considerazioni intorno all'etnografia visiva di Ernesto De Martino, in Clara Gallini / Francesco Faeta (a cura di), I viaggi nel sud di Ernesto De Martino. Fotografie di Arturo Zavattini, Franco Pinna e Ando Gilardi, Torino, Bollati Boringhieri, 1999, pp. 49-93.

Falaschi 1987 Giovanni Falaschi, Vittorini e la fotografia, in "AFT. Rivista di Storia e Fotografia", a. III, n. 5, 1987, pp. 34-40, in <http://rivista.aft.it/aftriv/controller. jsp?action=rivista_list> $(30.06 .2019)$.

Falqui 1954 Enrico Falqui, Il fotografo e il poeta, in “Tempo", 26 gennaio 1954.

G.P. 1954 G.P., Collaborazione e no tra uno scrittore e un fotografo, in "Milano sera", 6-7 febbraio 1954.

Il fotografo poeta 1954 Il fotografo poeta, in "Tempo", 12 marzo 1954.

Liva 2011 Walter Liva (a cura di), La fotografia e il neorealismo in Italia 1945-1965. Da Luigi Crocenzi al Gruppo Friulano per una nuova fotografia fino a Mario Giacomelli, Spilimbergo, CRAF, 2011. 
Liva 2019 Walter Liva, L'archivio fotografico di Luigi Crocenzi, in Alessandro

Giampaoli / Marco Andreani (a cura di), Mario Giacomelli, Giacomo Leopardi.

L'infinito, A Silvia, Cinisello Balsamo, Silvana, 2019, pp. 28-29.

Lupo 2011 Giuseppe Lupo, Vittorini Politecnico, Milano, Angeli, 2011.

Mazzacane 1996 Lello Mazzacane, Pinna e De Martino: una vicenda complessa, in Giuseppe Pinna / Maria Stefania Bruno / Claudio Domini / Giorgio Olmoti (a cura di), Franco Pinna. Fotografie 1944-1977, Milano, Federico Motta, 1996, pp. 125-135.

Orvieto 2005 Paolo Orvieto, Vittorini e I'"accostamento fotografico", in Anna Dolfi (a cura di), Letteratura e fotografia, Roma, Bulzoni, 2005, vol. II, pp. 61-82.

Paterlini 2014 Riccardo Paterlini, Conversazione illustrata. Contrabbando fototestuale in Elio Vittorini, in "Arabeschi", n. 4, luglio-dicembre 2014, pp. 125-140.

Pontillo 2018 Corinne Pontillo, "Conversazione in Sicilia" nella collana strenna Olivetti: storia di un'altra edizione illustrata, in Lorenzo Battistini et al. (a cura di), La letteratura italiana e le arti, Roma, ADI, 2018, in <http://www.italianisti.it/ upload/userfiles/files/PONTILLO\%20def.pdf> (30.06.2019).

Rizzarelli 2007 Maria Rizzarelli (a cura di), Elio Vittorini. Conversazione illustrata, Acireale-Roma, Bonanno, 2007.

Rizzarelli 2016 Maria Rizzarelli, “Che le parole salvino l'immagine”. Fotografia e narrazione in Vittorini, Pasolini e Sciascia, in Michele Cometa / Roberta Coglitore (a cura di), Fototesti. Letteratura e cultura visuale, Macerata, Quodlibet, 2016, pp. 205-223.

Russo 2011 Antonella Russo, Storia culturale della fotografia italiana, Torino, Einaudi, 2011.

Strand / Zavattini 1955 Paul Strand / Cesare Zavattini, Un paese, Torino, Einaudi, 1955.

Turrin 2003 Raffaella Turrin, Fotoracconti: il filo di una narrazione, in Amodeo / Giusa / Turrin 2003, pp. 17-28.

Van den Bossche / Baetens 2015 Bart Van den Bossche / Jan Baetens, Conversazioni istoriate. Intorno all'edizione illustrata di "Conversazione in Sicilia" 1953, in "Testo. Studi di teoria e storia della letteratura e della critica", a. XXXIV, n. 65, gennaio-giugno 2015, pp. 95-104.

Vittorini 1950a [1977] Elio Vittorini, Lettera a Giovanni Pirelli, 26 marzo 1950, in Vittorini 1977, p. 309.

Vittorini 1950b [1977] Elio Vittorini, Lettera a Giovanni Pirelli, 1 aprile 1950, in Vittorini 1977, p. 311.

Vittorini 1952 [2006] Elio Vittorini, Lettera ad Aldo Camerino, 5 dicembre 1952, in Vittorini 2006, p. 53.

Vittorini 1953a [2006] Elio Vittorini, Lettera a Leonardo Sciascia, 24 ottobre 1953, in Vittorini 2006, p. 123.

Vittorini 1953b Elio Vittorini, Conversazione in Sicilia, edizione illustrata a cura dell'autore con la collaborazione fotografica di Luigi Crocenzi, Milano, Bompiani, 1953.

Vittorini 1954 Elio Vittorini, La foto strizza l'occhio alla pagina, in "Cinema Nuovo", a. III, n. 33, 15 aprile 1954, pp. 200-202.

Vittorini 1955 [2006] Elio Vittorini, Lettera a Raul Lunardi, 3 luglio 1955, in Vittorini 2006, p. 289. 
Vittorini 1977 Elio Vittorini, Gli anni del «Politecnico». Lettere 1945-1951, a cura di Carlo Minoia, Torino, Einaudi, 1977.

Vittorini 2006 Elio Vittorini, Lettere 1952-1955, a cura di Edoardo Esposito e Carlo Minoia, Torino, Einaudi, 2006.

Zannier 1996 Italo Zannier (a cura di), Luigi Crocenzi: cultura della fotografia, Spilimbergo, CRAF, 1996.

\#Cintioli 1954 Giuseppe Cintioli, Lettera a Elio Vittorini del 10 febbraio 1954, lettera dattil., Milano, Centro Apice, Archivio Elio Vittorini (d'ora in poi MCAAEV), b. 4, Corrispondenza ricevuta, Cintioli Giuseppe.

\#Crocenzi s.d. Luigi Crocenzi, Conversazione. Risposte, lettera ms., s.d., Spilimbergo, CRAF, Archivio Luigi Crocenzi (d'ora in poi SCALC), Corrispondenza s-z, Vittorini Elio.

\#Crocenzi 1949 Luigi Crocenzi, Lettera a Elio Vittorini del 23 giugno 1949, lettera ms., MCAAEV, b. 4, Corrispondenza ricevuta, Crocenzi Luigi.

\#Crocenzi 1950 Luigi Crocenzi, Lettera a Elio Vittorini del 19 gennaio 1950, lettera ms., MCAAEV, b. 4, Corrispondenza ricevuta, Crocenzi Luigi.

\#Crocenzi 1953 Luigi Crocenzi, Lettera a Elio Vittorini del 21 settembre 1953, lettera ms., MCAAEV, b. 4, Corrispondenza ricevuta, Crocenzi Luigi.

\#Crocenzi 1954a Luigi Crocenzi, Lettera a Elio Vittorini del 10 gennaio 1954, lettera ms., MCAAEV, b. 4, Corrispondenza ricevuta, Crocenzi Luigi.

\#Crocenzi 1954b Luigi Crocenzi, Lettera a Valentino Bompiani, [s.d. ma tra l'11 e il 14 gennaio 1954], lettera dattil., MCAAEV, b. 11, f.2, sf. 2/11.

\#Crocenzi 1954c Luigi Crocenzi, Lettera a Elio Vittorini del 18 gennaio 1954, lettera ms., MCAAEV, b. 11, f.2, sf. 2/11.

\#Crocenzi 1954d Luigi Crocenzi, Lettera ad Angelo Del Boca del 29 gennaio 1954, lettera dattil., MCAAEV, b. 11, f.2, sf. 2/11.

\#Dania 1954 Luigi Dania, Lettera a Valentino Bompiani del 15 gennaio 1954, lettera dattil., MCAAEV, b. 11, f.2, sf. 2/11.

\#Falqui 1954a Enrico Falqui, Lettera a Elio Vittorini del 28 febbraio 1954, lettera dattil., MCAAEV, b. 5, Corrispondenza ricevuta, Falqui Enrico.

\#Falqui 1954b Enrico Falqui, Lettera a Elio Vittorini dell'11 marzo 1954, lettera dattil., MCAAEV, b. 5, Corrispondenza ricevuta, Falqui Enrico.

\#Nuove esperienze 1957 Nuove esperienze di illustrazione fotografica per “Conversazione in Sicilia" di Elio Vittorini, Fermo, CCF, 1957, materiale a stampa, SCALC, Corrispondenza s-z, Vittorini Elio. Riportato anche in Zannier 1996, p. 34.

\#Pirelli 1950 Giovanni Pirelli, Lettera a Elio Vittorini del 30 marzo 1950, lettera dattil., MCAAEV, b. 7, Corrispondenza ricevuta, Pirelli Giovanni. Riportata anche in Camerano / Crovi / Grasso 2007, vol I, pp. 147-150.

\#Pozzi Bellini 1950a Giacomo Pozzi Bellini, Lettera a Elio Vittorini del 20 marzo 1950, lettera ms., MCAAEV, b. 7, Corrispondenza ricevuta, Pozzi Bellini Giacomo.

\#Pozzi Bellini 1950b Giacomo Pozzi Bellini, Lettera a Elio Vittorini del 23 marzo 1950, lettera ms., MCAAEV, b. 7, Corrispondenza ricevuta, Pozzi Bellini Giacomo.

\#Vittorini 1954a Elio Vittorini, Lettera a Valentino Bompiani del 18 gennaio 1954, lettera dattil., MCAAEV, b. 1, Corrispondenza inviata, Bompiani Valentino. 
\#Vittorini 1954b Elio Vittorini, Lettera a Luigi Crocenzi, [s.d.] lettera dattil., MCAAEV, b. 1, Corrispondenza inviata, Crocenzi Luigi. (La lettera riporta sul frontespizio la data 15 gennaio 1954, ma non è di mano di Vittorini. Ė ragionevole pensare che la lettera sia stata scritta verso il 20 gennaio).

\#Vittorini 1954c Elio Vittorini, Lettera a Luigi Crocenzi del 20 gennaio 1954, minuta, MCAAEV, b. 1, Corrispondenza inviata, Crocenzi Luigi.

\#Vittorini 1954d Elio Vittorini, Lettera a Luigi Dania del 23 gennaio 1954, lettera dattil., MCAAEV, b. 1, Corrispondenza inviata, Bompiani Valentino.

\#Vittorini 1954e Elio Vittorini, Lettera a Valentino Bompiani del 26 gennaio 1954, lettera dattil., MCAAEV, b. 1, Corrispondenza inviata, Bompiani Valentino.

\#Vittorini 1956a Elio Vittorini, Lettera a Luigi Crocenzi e Giancarlo Silvetti del 16 febbraio 1956, lettera dattil., SCALC, Corrispondenza s-z, Vittorini Elio.

\#Vittorini 1956b Elio Vittorini, Lettera a Luigi Crocenzi del 5 dicembre 1956, lettera dattil., SCALC, Corrispondenza s-z, Vittorini Elio.

\#Vittorini 1957 Elio Vittorini, Lettera a Luigi Crocenzi del 22 gennaio 1957, lettera dattil., SCALC, Corrispondenza s-z, Vittorini Elio. 